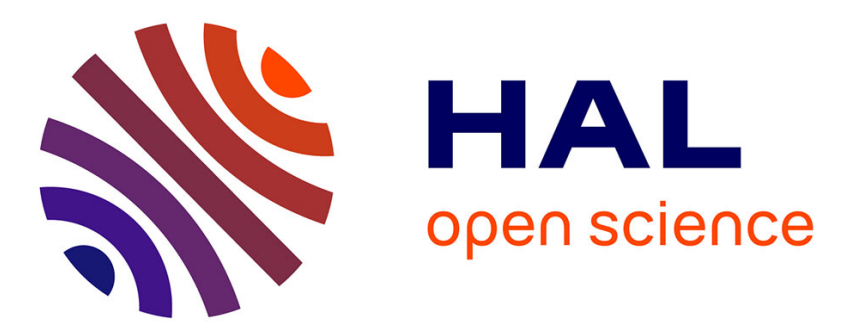

\title{
Sampled-Data Adaptive Observer For a Class of State Affine Output-Injection Nonlinear Systems
}

\author{
T. Folin, Tarek Hamed-Ali, Fouad Giri, Laurent Burlion, Françoise
}

Lamnabhi-Lagarrigue

\section{- To cite this version:}

T. Folin, Tarek Hamed-Ali, Fouad Giri, Laurent Burlion, Françoise Lamnabhi-Lagarrigue. SampledData Adaptive Observer For a Class of State Affine Output-Injection Nonlinear Systems. IEEE Transactions on Automatic Control, 2016, 61 (2), pp.462-467. 10.1109/TAC.2015.2437522 . hal01260152

\section{HAL Id: hal-01260152}

https://hal-centralesupelec.archives-ouvertes.fr/hal-01260152

Submitted on 13 Jul 2020

HAL is a multi-disciplinary open access archive for the deposit and dissemination of scientific research documents, whether they are published or not. The documents may come from teaching and research institutions in France or abroad, or from public or private research centers.
L'archive ouverte pluridisciplinaire HAL, est destinée au dépôt et à la diffusion de documents scientifiques de niveau recherche, publiés ou non, émanant des établissements d'enseignement et de recherche français ou étrangers, des laboratoires publics ou privés. 


\title{
Sampled-Data Adaptive Observer For a Class of State-Affine Output-Injection Nonlinear Systems
}

\author{
T. Folin, T. Ahmed-Ali, F. Giri, L. Burlion, F. Lamnabhi-Lagarrigue
}

\begin{abstract}
The problem of observer design is addressed for outputinjection nonlinear systems. A major difficulty with this class of systems is that the state equation involves an output-dependent term that is explicitly dependent on unknown parameters. As the output is only accessible to measurement at sampling times, the outputdependent term turns out to be (almost all time) subject to a double uncertainty, making previous adaptive observers inappropriate. Presently, a new hybrid adaptive observer is designed and shown to be exponentially convergent under ad-hoc conditions.
\end{abstract}

Index Terms - Adaptive observer, Sampled-data nonlinear systems

\section{INTRODUCTION}

Designing adaptive observers for nonlinear systems has been given a great deal of interest since the late eighties $([2,3,4,7,8])$. Several design methods for obtaining exponentially convergent adaptive observers have been developed. However, almost all these design methods provide continuous-time adaptive observers that need discretization for practical implementation purpose. The point is that exact discretization is a highly complex issue due to the strong nonlinearity of the adaptive observer. On the other hand, there is no guarantee that approximate discrete-time versions can preserve the performances of the original continuous-time adaptive observers. This explains why quite a few studies have, so far, focused on designing sampled-data adaptive observers that apply to nonlinear systems subject to parametric uncertainty. A first attempt has been made in [1] where an adaptive observer, operating according to the continuousdiscrete design principle, has been developed for a class of state affine system with unknown parameters. Later on, an adaptive observer has been developed in [5], following the hybrid-observer design principle.

A common limitation of the (sampled-output) nonlinear adaptive observers of [1,5] is that, they are not applicable to output-injection systems. In these systems, the output signal is feed back through the state equation. In the context of adaptive observers, the outputdependent terms (entering the state equation) are also dependent on unknown parameters. As the output measurements are missing (except at sampling times), it turns out that those output-dependent terms are subject to a double uncertainty (parametric and output). This is a challenging difficulty that existing adaptive observers can not cope with. Presently, a new nonlinear adaptive observer is designed by combining ideas from continuous-time adaptive observers (e.g. $[3,8]$ ) and from continuous-discrete hybrid adaptive observers [6]. Accordingly, the proposed observer includes an adaptive state estimator, an adaptation parameter law, and an inter-sample outputestimator. The latter is an instrumental component as the output estimation error is used to correct the trajectories of the state and the parameter estimates. It involves state and parameter projections and undergoes a periodic resetting which makes it the only discontinuous part of the observer. It is formally established that the adaptive observer is exponentially convergent provided the sampling period is small and a persistent excitation condition holds. The fact that the state and parameter estimate trajectories are continuous is another appealing feature of the observer.

T. Folin, T. Ahmed-Ali and F. Giri are with the GREYC Lab, University of Caen Basse-Normandie, ENSICAEN, 14032 Caen, France. T. Folin is also with BodyCap SAS, 14200 Hérouville Saint Clair, France.

L. Burlion is with ONERA, the French Aerospace Lab, BP 74025, 31055 Toulouse, France.

F. Lamnabhi-Lagarrigue is with the LSS-CNRS, SUPELEC, EECI, 91192 Gif-sur-Yvette, France
The paper is organized as follows: the class of systems dealt with is described along with the proposed observer in Section 2; the main theorem describing the observer performances is presented in Section 3 ; all technical proofs are appended.

\section{Class of Systems AND AdAPTIVE OBSERVER}

The system under study is described by the following model:

$\dot{x}(t)=A_{0} x(t)+\psi_{0}(u(t), y(t)) \theta_{0}+\psi_{1}(u(t)) \theta_{1}$

$y\left(t_{k}\right)=c x\left(t_{k}\right)$

with,

$A_{0} \in \mathbf{R}^{n \times n} ; c \in \mathbf{R}^{1 \times n}, \theta_{0} \in \mathbf{R}^{p}, \quad \theta_{1} \in \mathbf{R}^{m}$

$\psi_{0}(u, y) \in \mathbf{R}^{n \times p} ; \psi_{1}(u) \in \mathbf{R}^{n \times m}$,

where $u$ and $y$ denote the system input and output, respectively; $x \in \mathbf{R}^{n}$ is the state vector, and the pair $\left(A_{0}, c\right)$ is known and detectable. The system order $n$ is known but the parameter vectors $\theta_{0}$ and $\theta_{1}$ are not. The function $\psi_{0}(.,$.$) is class C^{1}$ while $\psi_{1}($.$) is$ just continuous. The input signal $u$ is bounded and the mapping $u \rightarrow x$ (defined by equation (1a)) is $L_{\infty}$-stable. Then, it readily follows that, in turn the state $x$ and the output $y$ are bounded. Note that signal boundedness is a usual assumption in the literature of nonlinear observers. The output equation (1b) emphasizes the fact that the signal $y$ is only accessible to measurements at sampling instants $t_{k}$. The latter is any increasing sequence so that $t_{k} \rightarrow \infty$ as $k \rightarrow \infty$. As the state equation (1a) involves $y$, through $\psi_{0}(u, y)$, the system (1a-b) is said output-injection type [3]. A large class of practical systems fit the model (1a) and so can be observed using the adaptive observer proposed hereafter. Among these systems, Lorenz chaotic model is of the form (1a) with $u(t)=0$ and $\theta_{1}=0$, and Duffing oscillator model which involves the specific input $u(t)=\cos (\omega t)$ [4].

The problem at hand consists in designing an observer that provides accurate online estimates of the state $x(t)$ and the parameter vectors $\theta_{0}$ and $\theta_{1}$. State and parameter estimation must only rely on the input signal $u(t)$ and the sampled output measurements $y\left(t_{k}\right)$. A major difficulty in this problem is that the system involves two unknown parameter vectors with different properties. The parameter vector $\theta_{1}$ comes linearly in the state equation (1a), while $\theta_{0}$ does not. Indeed, the output $y$ in $\psi_{0}(u, y)$ is only accessible to measurement at sampling times. That is, the term $\psi_{0}(u(t), y(t)) \theta_{0}$ in (1a) is all time subject to a double uncertainty, except at sampling times. This double uncertainty makes currently existing sampled-data adaptive observers inappropriate for the system $(1 \mathrm{a}-\mathrm{b})$. Indeed, those proposed in $[1,5]$ only apply to state-affine systems (in which case $\theta_{0}=0$ ). As no existing observer is applicable to the system (1a-b), the following sampled-output adaptive observer is presently proposed:

$$
\begin{aligned}
\dot{\hat{x}}(t)= & A_{0} \hat{x}(t)+\left[\psi_{0}(u(t), \operatorname{sat}(z(t))) \psi_{1}(u(t))\right] \hat{\theta}(t) \\
& -K(c \hat{x}(t)-z(t))+\lambda(t) \dot{\hat{\theta}}(t) \\
\dot{\hat{\theta}}(t)= & -R(t) \lambda^{T}(t) c^{T}(c \hat{x}(t)-z(t)), \text { with } \lambda(t) \in \mathbf{R}^{n \times(m+p)} \\
\hat{\theta}(t)= & {\left[\hat{\theta}_{0}^{T}(t) \hat{\theta}_{1}^{T}(t)\right]^{T}, \text { with } \hat{\theta}_{0}(t) \in \mathbf{R}^{p}, \hat{\theta}_{1}(t) \in \mathbf{R}^{m} } \\
\dot{z}(t)= & c A_{0} P_{x}(\hat{x}(t))+c\left[\psi_{0}(u(t), \operatorname{sat}(z(t))) \psi_{1}(u(t))\right] P_{\theta}(\hat{\theta}(t)), \\
& \text { for } t \in\left(t_{k}, t_{k+1}\right)
\end{aligned}
$$


$z\left(t_{k}\right)=y\left(t_{k}\right)$

$\dot{\lambda}(t)=\left(A_{0}-K c\right) \lambda(t)+\left[\psi_{0}(u(t), \operatorname{sat}(z(t))) \psi_{1}(u(t))\right]$

$\dot{R}=R-R \lambda^{T} c^{T} c \lambda R$, with $R(0)=I_{(m+p) \times(m+p)}$

where the observer gain $K \in \mathbf{R}^{n}$ is chosen so that the matrix $A_{0}-K c$ is Hurwitz; $P_{x}$ and $P_{\theta}$ denote projection functions, respectively on $\Omega_{x} \subset \mathbf{R}^{n}$ and $\Omega_{\theta} \subset \mathbf{R}^{m+p}$, the latter being the balls centered on the origin, with known radiuses $\rho_{x}$ and $\rho_{\theta}$, such that:

$\theta \in \Omega_{\theta}$ and $x(t) \in \Omega_{x}, \forall t \geq 0$

For convenience, the definitions of projection and saturation functions are recalled:

$$
\begin{gathered}
P_{x}(w)=\min \left(\|w\|, \rho_{x}\right) \frac{w}{\|w\|}, \quad P_{\theta}(w)=\min \left(\|w\|, \rho_{\theta}\right) \frac{w}{\|w\|}, \\
\text { and } \operatorname{sat}(z)=\operatorname{sgn}(z) \min \left(|z|, y_{M}\right)
\end{gathered}
$$

where $y_{M}>0$ is any upper bound of output i.e.

$$
y_{M} \geq \sup _{0 \leq t<\infty}|y(t)|
$$

where $\operatorname{sgn}(z)$ designates the sign function. All initial values $(\hat{\theta}(0), \hat{x}(0) \ldots)$ in $(3 \mathrm{a}-\mathrm{g})$ are allowed to be arbitrarily chosen (because to the observer will prove to be globally convergent). In view of ( $3 \mathrm{~d}-$ e), the variable $z(t)$ is an estimate of the output $y(t)$ over the open interval $\left(t_{k}, t_{k+1}\right)$, given the output samples $y\left(t_{k}\right), y\left(t_{k-1}\right) \ldots$ The output estimator (3d-e) is resorted to cope with the sampled nature of output measurements. Also, the output estimation error acts as driving input of both the state estimation equation (3a) and the parameter adaptation law (3b). The model component $\psi_{0}(u, y)$ which is outputdependent is replaced in the observer by the "reconstructed signal" $\psi_{0}(u(t), \operatorname{sat}(z(t)))$. This is coherent with the fact that $z(t)$ is an estimate of $y(t)$. The saturation of $z(t)$ is used to cope with the fact that, at this stage, this signal is not yet proved to be bounded. Without using that saturation, it will not be possible to ensure the boundedness of the adaptation gain $\lambda$, nor that of the remaining observer signals. Note that the presence of the quantity $\psi_{0}(u(t)$, sat $(z(t)))$ is precisely what makes (3a-g) fundamentally different from previous (sampleddata) adaptive observers e.g. those in $[1,5]$.

Remark 1. As $\psi_{0}(.,$.$) and \psi_{1}(.,$.$) are continuous and A_{0}-K c$ is Hurwitz, two real scalars $\psi_{M}>0$ and $\lambda_{M}>0$ can be found such that: $\sup _{t}\left\|\left[\psi_{0}(u(t), \operatorname{sat}(z(t))) \psi_{1}(u(t))\right]\right\| \leq \psi_{M}, \quad \sup _{t}\|\lambda(t)\| \leq \lambda_{M}$

\section{ADAPTIVE OBSERVER ANALYSIS}

The adaptive observer defined by equations (3a-g) will now be analyzed. As pointed out earlier, a major difficulty in the analysis is to deal with the term $\psi_{0}(u(t), y(t)) \theta_{0}$ (in equation (1a)) which entails a double uncertainty (a parametric uncertainty on $\theta_{0}$ and measurement uncertainty on the output $y(t))$. Another difficulty comes from the hybrid (continuous-discrete) nature of the output estimator (3d-e) of the observer. For analysis purpose, the following notations are introduced:

$\tilde{x}=\hat{x}-x, \quad e=z-y, \quad \tilde{\theta}=\hat{\theta}-\theta, \quad \theta=\left[\begin{array}{ll}\theta_{0}^{T} & \theta_{1}^{T}\end{array}\right]^{T}, \tilde{\theta}=\left[\begin{array}{ll}\tilde{\theta}_{0}^{T} & \tilde{\theta}_{1}^{T}\end{array}\right]^{T}$

where the vector partitions in (7) match those in (3c). Also, introduce the following augmented error:

$\eta=\tilde{x}-\lambda \tilde{\theta}$

This error undergoes the following equation (proof in Appendix A):

$\dot{\eta}=\left(A_{0}-K c\right) \eta+K e+\left(\psi_{0}(u, \operatorname{sat}(z))-\psi_{0}(u, y)\right) \theta_{0}$
This is completed with similar equations, obtained using (7), (3a-g) and (1a-b), describing the dynamics of the errors $e$ and $\tilde{\theta}$ :

$$
\begin{aligned}
& \dot{\tilde{\theta}}=-R \lambda^{T} c^{T}(c \lambda \tilde{\theta}+c \eta-e) \\
& \dot{e}=c A_{0}\left(P_{x}(\hat{x})-P_{x}(x)\right)+c\left[\psi_{0}(u, \operatorname{sat}(z)) \psi_{1}(u)\right]\left(P_{\theta}(\hat{\theta})-P_{\theta}(\theta)\right) \\
& \quad+c\left[\psi_{0}(u, \operatorname{sat}(z))-\psi_{0}(u, \operatorname{sat}(y)) 0\right] P_{\theta}(\theta), \quad t \in\left[t_{k}, t_{k+1}\right) \\
& e\left(t_{k}\right)=0
\end{aligned}
$$

where the fact that $x=P_{x}(x)$ has been used in (9c). The analysis of the error system (9a-d) will now be accomplished, taking benefit from the fact that the time-varying matrix $R$ (solution of $(3 \mathrm{~g})$ ) is bounded symmetric positive definite, provided the following persistent excitation (PE) condition holds (e.g. [4,8]): $\exists \varepsilon_{0}, \varepsilon_{1}>0, \exists \delta>0, \forall t>0$ :

$$
\varepsilon_{0} I_{(m+p) \times(m+p)}<\int_{t}^{t+\delta} \lambda^{T}(s) c^{T} c \lambda(s) d s<\varepsilon_{1} I_{(m+p) \times(m+p)}
$$

Under this condition, it is readily checked that the matrix inverse $R^{-1}$, which also is bounded positive definite, undergoes the equation $d\left(R^{-1}\right) / d t=-R^{-1}+\lambda^{T} c^{T} c \lambda$. Then, two positive real numbers $(r, \bar{r})$ would exist such that, for all $t \geq 0$ :

$r I_{(m+p) \times(m+p)} \leq R^{-1}(t)=\left(R^{-1}(t)\right)^{T} \leq \bar{r} I_{(m+p) \times(m+p)}$

The point with condition (10) is that it involves the vector signal $\lambda(t)$ which, in view of (3d) and (3f), depends on the observer signals and parameters (i.e. $\left.z(t), \hat{x}(t), \hat{\theta}_{0}(t), \hat{\theta}_{1}(t)\right)$. A convenient $\mathrm{PE}$ condition is one that only depends on the system (not the observer) signals and parameters. This issue is coped with in the following lemma, the proof of which is placed in Appendix B:

Lemma 1. Let $\lambda^{*}(t)$ denotes the solution of the equation,

$\dot{\lambda}^{*}(t)=\left(A_{0}-K c\right) \lambda^{*}(t)+\left[\psi_{0}(u(t), y(t)) \psi_{1}(u(t))\right]$

with $\lambda^{*}(0)=\lambda(0)$, and introduce the definition,

$\tau=\sup _{k}\left(t_{k}-t_{k-1}\right)$.

Suppose the following PE property is holding:

$\exists \bar{\varepsilon}_{0}, \bar{\varepsilon}_{1}>0, \exists \bar{\delta}>0, \forall t>0$ :

$\bar{\varepsilon}_{0} I_{(m+p) \times(m+p)}<\int_{t}^{t+\delta} \lambda^{* T}(s) c^{T} c \lambda^{*}(s) d s<\bar{\varepsilon}_{1} I_{(m+p) \times(m+p)}$

Then, there exists a real constant $\tau_{0}>0$ such that, if $0<\tau \leq \tau_{0}$ then, in turn property (10) holds

Remark 2. a) Expressing the PE condition (14) in terms of the matrix gain $\lambda$ is a usual practice in the literature, e.g. $[1,3,4,5,8]$.

b) An explicit description of how small $\tau_{0}$ is provided in the proof of Lemma 1. In the sequel, the (system-dependent) PE condition (14) will be supposed to be true and the maximal sampling period is assumed to be sufficiently small (in the sense that $0<\tau \leq \tau_{0}$ ). Then, by Lemma 1, the (observer-dependant) PE property (10) and the matrix inequalities (11) can be used throughout the paper

Making use of (11), the following Lyapunov function is considered:

$V=\tilde{\theta}^{T} R^{-1} \tilde{\theta}+\eta^{T} P \eta$

where $P=P^{T}$ is any positive definite matrix satisfying the inequality:

$P\left(A_{0}-K c\right)+\left(A_{0}-K c\right)^{T} P \leq-\mu I$

and $\mu$ is any positive constant such that:

$\mu>\frac{4 \lambda_{M}^{2}\|c\|^{4}}{\alpha}$ 
where $\lambda_{M}$ is as in (6) and $\alpha$ is any positive real scalar such that:

$r \geq 2 \alpha$

where $r$ is as in (11). Let $\tau^{*}>0$ denote the largest scalar satisfying:

$\tau^{*} \leq \tau_{0}, \tau^{*} e^{\sigma \tau^{*} / 2}<\frac{1}{\|c\|\left\|\theta_{0}\right\| \psi_{M}^{*}}, \frac{\tau^{*} e^{\sigma \tau^{*} / 2}}{1-\|c\|\left\|\theta_{0}\right\| \psi_{M}^{*} \tau^{*} e^{\sigma \tau^{*} / 2}}<\frac{1}{\gamma M}$

where $\sigma$ is any scalar such that $0<\sigma<\frac{\sigma_{0}}{2}$ and

$\psi_{M}^{*}=\sup _{|u| \leq u_{M},|y| \leq y_{M}}\left\|\frac{\partial}{\partial y} \psi_{0}(u, y)\right\|$, with $u_{M} \stackrel{\text { def }}{=} \sup _{0 \leq t<\infty}|u(t)|$

$M=\max \left(\left\|c A_{0}\right\|,\left\|c A_{0}\right\| \lambda_{M},\|c\| \psi_{M}\right), \quad \gamma=\sqrt{\frac{2 \sigma_{1}}{\sigma_{0} \sigma_{2}}}$

$\sigma_{0}=\min \left\{\frac{1}{2}, \frac{1}{\lambda_{\max }(P)}\left(\frac{\mu}{2}-\frac{2 \lambda_{M}^{2}\|c\|^{4}}{\alpha}\right)\right\}$,

and $\sigma_{1}=\frac{2}{\mu}\|P\|^{2}\left(\|K\|^{2}+\rho_{\theta}^{2}\left(\psi_{M}^{*}\right)^{2}\right)+\frac{2 \lambda_{M}^{2}}{\alpha}\|c\|^{2}$

$\sigma_{2}=\inf \left\{\lambda_{\text {min }}(P), r\right\}$

where $\lambda_{\text {max }}($.$) and \lambda_{\text {min }}($.$) are the maximum and minimum$ eigenvalues. Then, one has the following main result in Appendix C.

Theorem 1. Let the sampled-output adaptive observer (3a-g) be applied to the system (1a-b). Suppose that the sampling period satisfies $\tau<\tau^{*}$ and that the PE condition (12) holds. Then:

$$
\left\|\left[\begin{array}{c}
\tilde{\theta}(t) \\
\eta(t)
\end{array}\right]\right\| \leq \frac{1-\|c\|\left\|\theta_{0}\right\| \psi_{M}^{*} \tau e^{\sigma \tau / 2}}{1-\|c\|\left\|\theta_{0}\right\| \psi_{M}^{*} \tau e^{\sigma \tau / 2}-\gamma M \tau e^{\sigma \tau / 2}} e^{-\sigma / 2}, \forall t \geq t_{0}
$$

Remark 3. a) By Theorem 1 , both $\tilde{\theta}(t)$ and $\eta(t)$ exponentially tends to zero. On the other, (8) entails $\|\tilde{x}\|=\|\eta+\lambda \tilde{\theta}\| \leq\|\eta\|+\|\lambda\|\|\tilde{\theta}\|$ and $\|\lambda\|$ is bounded by (6). It follows that $\|\tilde{x}(t)\|$ is exponentially vanishing.

b) Notice that inequalities (16), (17), and (18) define a feasible algebraic problem. Indeed, the constant observer gain $K$ is first chosen so that $\left(A_{0}-K c\right)$ is Hurwitz. Then, (3f) shows that $\lambda$ and its bound $\lambda_{M}$ in (6) are entirely dependent on the choice of $K$. Then, equation $(3 \mathrm{~g})$ implies that in turn $R$, and so its bounds $r$ and $\bar{r}$ in (18), are dependent on the choice of $K$. Owing to $\bar{r}$, it is readily checked using (11) that a possible value is $r=e^{-\delta} \varepsilon_{0}$ (see e.g. [4]).

Then, (18) implies that, in turn $\alpha$ is dependent on $\left(\delta, \varepsilon_{0}\right)$ (which themselves are dependent on $K$ ) and, due to (17), so is $\mu$. Finally, inequality (16) shows that $P$ depends on $\delta$ and $\varepsilon_{0}$

\section{Simulation}

To illustrate the performances of the adaptive observer, we are considering the following example of the form (1a) with,

$A_{0}=\left[\begin{array}{cc}0 & 1 \\ -1 & -1\end{array}\right], \psi_{0}(u(t), y(t))=\left[\begin{array}{c}\cos (y(t)) \\ \sin (y(t))\end{array}\right], \psi_{1}(u(t))=\left[\begin{array}{c}0 \\ u(t)\end{array}\right]$,

$c=[10], \quad \theta_{0}=-4.5, \quad \theta_{1}=1.5, \quad$ and initial conditions $x_{1}(0)=4.7, x_{2}(0)=0.1$. It is readily checked that the autonomous system (with $u(t)=0$ ) has an equilibrium at $(0,0)$ and its linear approximation (around the equilibrium) has complex poles. That is, the system is of oscillating nature). The observer gain $K$ is selected so that the two eigenvalues of $A_{0}-K c$ take the values -1.01 and -1.00 . It turns out that $K=[1.01,-1]$. The parameter and signal bounds involved (5a-b) are set to $\rho_{\theta}=\rho_{x}=100$, and $y_{M}=15.7$. It is checked by simulation that, the last two bounds hold whenever the system (1a-b) is excited by square periodic inputs $u(t)$ with amplitude not larger than 5 . An example of such input signals is shown, together with the corresponding system output, in Fig. 1 and both are used in the observer (3a-g). The sampling period is set to a constant value $\tau=0.5 \mathrm{~s}$. Then, the quality of the obtained parameter and state estimates is illustrated by Figs 3 and 4. Because of space limitation, only estimation errors are provided. The figures show a quite satisfactory estimation quality confirming Theorem 1 .

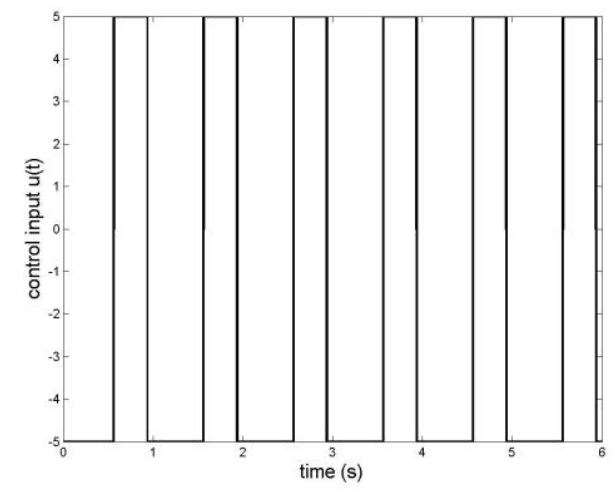

Fig. 1. Input signal.
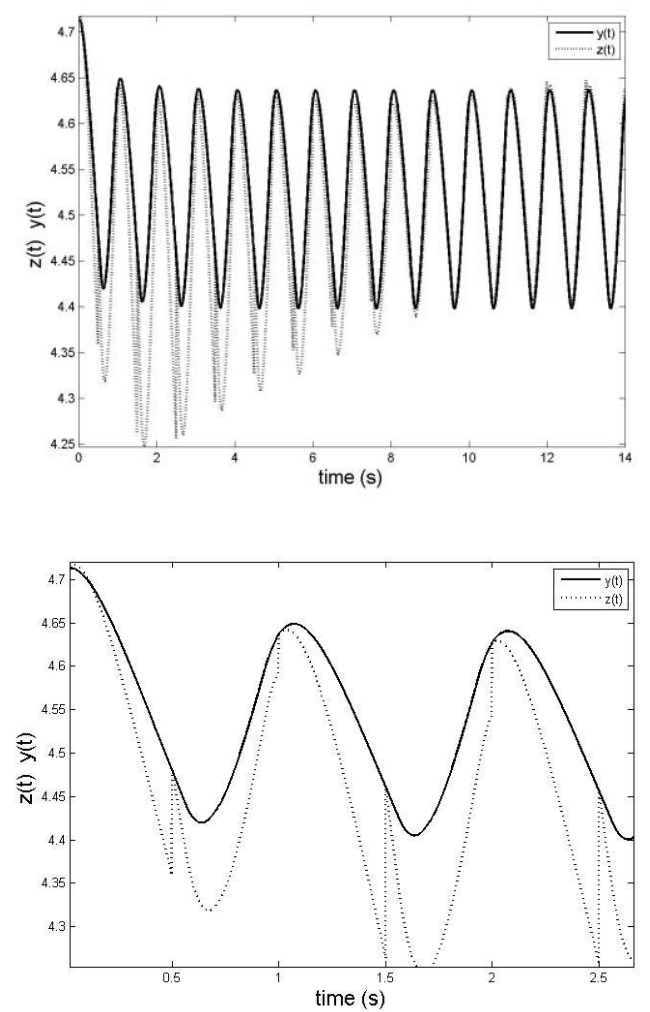

Fig. 2. Top: Illustration of perfect matching between the system output $y(t)$ (solid) and predicted output $z(t)$ (dotted). Bottom: zoom on the true and predicted outputs during the transient stage.

\section{CONCLUDING REMARKS}

This study has addressed the problem of adaptively estimating the state and the parameters of output-injection systems of the form (1a-b), based on sampled-output data. A major difficulty is that, the outputdependent term $\psi_{0}(u, y) \theta_{0}$ (entering the state equation) is subject to both parametric and output uncertainties. This makes existing sampled-data adaptive observers inappropriate for the systems (1a-b). 
Theorem 1 shows that the new hybrid adaptive observer (3a-g) is suitable for these systems as it enjoys exponential convergence under ad-hoc conditions, including the sampling period smallness and the (system-dependent) PE condition of Lemma 1. Another feature is that the trajectory of the state estimate $\hat{x}(t)$ is continuous (only the output estimation $z(t)$ is discontinuous).
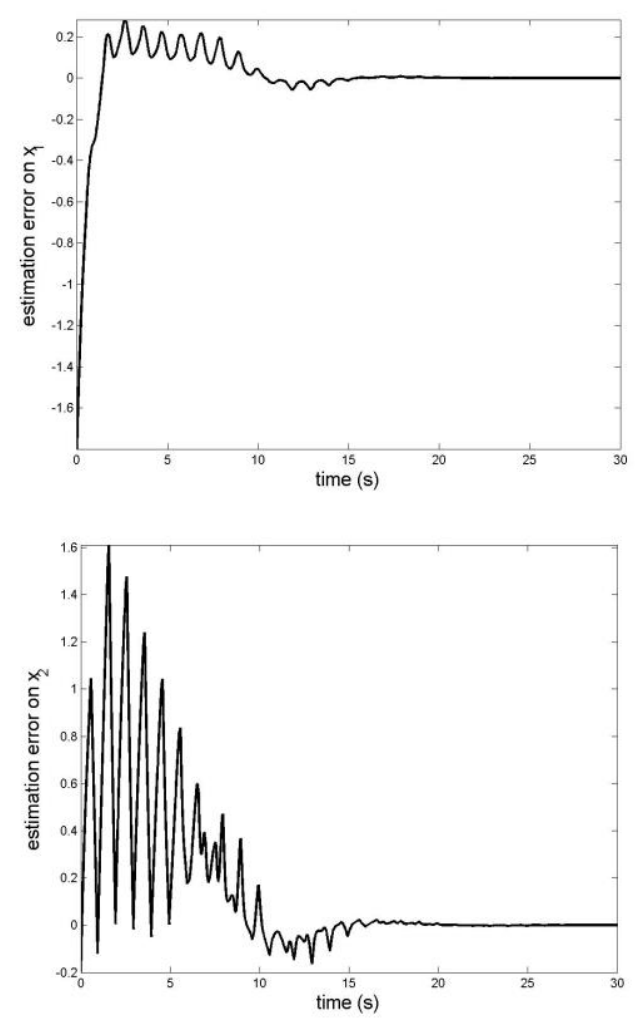

Fig. 3. Estimation errors $\tilde{x}_{1}=\hat{x}_{1}-x_{1}$ (top) and $\tilde{x}_{2}=\hat{x}_{2}-x_{2}$ (bottom).
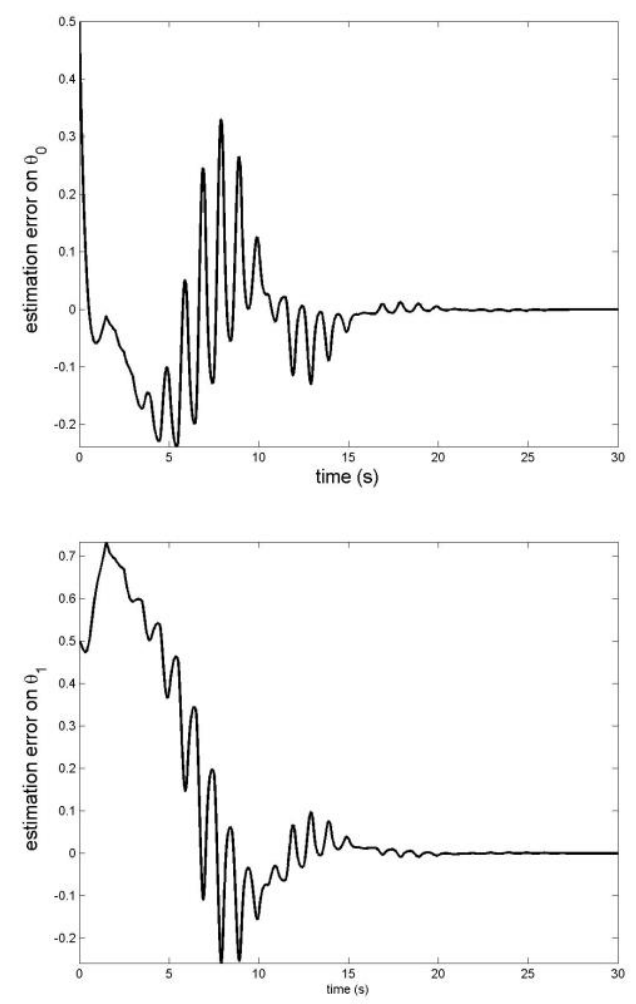

Fig. 4. Estimation errors $\tilde{\theta}_{0}=\hat{\theta}_{0}-\theta_{0}$ (top) and $\tilde{\theta}_{1}=\hat{\theta}_{1}-\theta_{1}$ (bottom).
APPENDIX A. PROOF OF EQUATION (9A)

Subtracting (1a) from (3a), one gets using (7):

$$
\begin{aligned}
\dot{\tilde{x}}= & A_{0} \tilde{x}+\psi_{0}(u, \operatorname{sat}(z)) \hat{\theta}_{0}-\psi_{0}(u, y) \theta_{0}+\psi_{1}(u) \tilde{\theta}_{1}-K(c \hat{x}-z)+\lambda \dot{\hat{\theta}} \\
= & \left(A_{0}-K c\right) \tilde{x}+\psi_{0}(u, \operatorname{sat}(z)) \tilde{\theta}_{0}+\left(\psi_{0}(u, \operatorname{sat}(z))-\psi_{0}(u, y)\right) \theta_{0} \\
& +\psi_{1}(u) \tilde{\theta}_{1}+K e+\lambda \dot{\hat{\theta}}
\end{aligned}
$$

By (8), $\eta+\lambda \tilde{\theta}$ is substituted to $\tilde{x}$ on the right side of (A1), yielding:

$$
\begin{aligned}
\dot{\tilde{x}}=( & \left.A_{0}-K c\right) \eta+\psi_{0}(u, \operatorname{sat}(z)) \tilde{\theta}_{0}+\left(\psi_{0}(u, \operatorname{sat}(z))-\psi_{0}(u, y)\right) \theta_{0} \\
& +\psi_{1}(u) \tilde{\theta}_{1}+K e+\lambda \dot{\hat{\theta}}+\left(A_{0}-K c\right) \lambda \tilde{\theta}
\end{aligned}
$$

From (A2) and (8), one gets deriving $\eta$ :

$$
\begin{aligned}
\dot{\eta}=\dot{\tilde{x}} & -\lambda \dot{\tilde{\theta}}-\dot{\lambda} \tilde{\theta}=\left(A_{0}-K c\right) \eta+\psi_{0}(u, \operatorname{sat}(z)) \tilde{\theta}_{0} \\
& +\left(\psi_{0}(u, \operatorname{sat}(z))-\psi_{0}(u, y)\right) \theta_{0} \\
& +\psi_{1}(u) \tilde{\theta}_{1}+K e+\left(A_{0}-K c\right) \lambda \tilde{\theta}-\dot{\lambda} \tilde{\theta}
\end{aligned}
$$

Using (3f), (A3) simplifies to:

$\dot{\eta}=\left(A_{0}-K c\right) \eta+K e+\left(\psi_{0}(u, \operatorname{sat}(z))-\psi_{0}(u, y)\right) \theta_{0}$

which establishes $(9 \mathrm{a})$

\section{ApPendix B. ProOf OF LEMMA 1.}

Letting $\tilde{\lambda}(t)=\lambda(t)-\lambda^{*}(t)$, it follows subtracting (3f) from (12) that: $\dot{\tilde{\lambda}}(t)=\left(A_{0}-K c\right) \tilde{\lambda}(t)+\left[\psi_{0}(u(t), \operatorname{sat}(z(t)))-\psi_{0}(u(t), y(t)) 0\right] \quad(\mathrm{B} 1)$ with $\tilde{\lambda}(0)=0$ (because $\left.\lambda^{*}(0)=\lambda(0)\right)$. As $\psi_{0}$ is continuous, the last term on the right side of (B1) is bounded as follows:

$$
\begin{aligned}
\left\|\psi_{0}(u, \operatorname{sat}(z))-\psi_{0}(u, y)\right\| & \leq \psi_{M}^{*}|\operatorname{sat}(z)-y| \\
& \leq \psi_{M}^{*}|\operatorname{sat}(z)-\operatorname{sat}(y)| \leq \psi_{M}^{*}|e|
\end{aligned}
$$

because $\operatorname{sat}(y)=y$, with $\psi_{M}^{*}$ as in (20). As $A_{0}-K c$ is Hurwitz, it follows from (B1) and (B2) that:

$\sup _{0 \leq s \leq t}\|\tilde{\lambda}(s)\| \leq K_{\lambda} \psi_{M}^{*} \sup _{0 \leq s \leq t}|e(s)|$

using $\tilde{\lambda}(0)=0$, where $K_{\lambda}=\int_{0}^{\infty}\left\|e^{\left(A_{0}-K c\right) s}\right\| d s$. Note that $K_{\lambda}$ is finite and can a priori be determined because $A_{0}-K c$ is known. On the other hand, equation (3d) gives, for all $t \in\left[t_{k}, t_{k+1}\right)$ :

$$
\begin{aligned}
z(t)= & y\left(t_{k}\right)+\int_{t_{k}}^{t} c A_{0} P_{x}(\hat{x}(s)) d s \\
& +\int_{t_{k}}^{t} c\left[\psi_{0}(u(s), \operatorname{sat}(z(s))) \psi_{1}(u(s))\right] P_{\theta}(\hat{\theta}(s)) d s
\end{aligned}
$$

Recall that the output $y(t)$ undergoes an equation like (3d) replacing there all estimates by their true values. Then, an expression similar to (B4) is obtained for $y(t)$, i.e. for all $t \in\left[t_{k}, t_{k+1}\right)$ :

$$
y(t)=y\left(t_{k}\right)+\int_{t_{k}}^{t} c A_{0} x(s)+c\left[\psi_{0}(u(s), y(s)) \psi_{1}(u(s))\right] \theta d s
$$

Subtracting (B5) from (B4), one gets for all $t \in\left[t_{k}, t_{k+1}\right)$ :

$$
\begin{aligned}
e(t)= & z(t)-y(t)=\int_{t_{k}}^{t} c A_{0}\left(P_{x}(\hat{x}(s))-x(s)\right) d s \\
& +\int_{t_{k}}^{t} c\left[\psi_{0}(u(s), \operatorname{sat}(z(s))) \psi_{1}(u(s))\right] P_{\theta}(\hat{\theta}(s)) d s \\
& -\int_{t_{k}}^{t} c\left[\psi_{0}(u(s), y(s)) \psi_{1}(u(s))\right] \theta d s
\end{aligned}
$$

The terms under the integral symbols involve both system signals (i.e. $u, x, y$ ) and observer signals (i.e. $\hat{x}, \hat{\theta}, z$ ). The former are bounded by assumption. The latter come in through the projection functions. It turns out that, all terms under integrals are bounded. Then, it follows from (B6) that, there exists a real $0<K_{e}<\infty$ such that:

$|e(t)|=|z(t)-y(t)| \leq K_{e} \tau$, , for all $t \in\left[t_{k}, t_{k+1}\right)$ 
$\sup _{0 \leq s \leq t}\|\tilde{\lambda}(s)\| \leq K_{\lambda} \psi_{M}^{*} K_{e} \tau$

On the other hand, it is readily checked that:

$\int_{t}^{t+\delta} \lambda^{T}(s) c^{T} c \lambda(s) d s$

$=\int_{t}^{t+\delta} \lambda^{* T}(s) c^{T} c \lambda^{*}(s) d s+2 \int_{t}^{t+\delta} \lambda^{* T}(s) c^{T} c \tilde{\lambda}(s) d s+\int_{t}^{t+\delta} \tilde{\lambda}^{T}(s) c^{T} c \tilde{\lambda}(s) d s$

$\geq \int_{t}^{t+\delta} \lambda^{* T}(s) c^{T} c \lambda^{*}(s) d s-2 \lambda_{M}\|c\|^{2}\left(\int_{t}^{t+\delta}\|\tilde{\lambda}(s)\| d s\right) I_{(m+p) \times(m+p)}$

using (6) and (2b). Combining (B9) and (B8) gives, using (14):

$\int_{t}^{t+\delta} \lambda^{T}(s) c^{T} c \lambda(s) d s \geq \bar{\varepsilon}_{0} I_{(m+p) \times(m+p)}$

$$
-2 \tau \lambda_{M} K_{\lambda} \psi_{M}^{*} K_{e} \delta\|c\|^{2} I_{(m+p) \times(m+p)}
$$

This establishes the right side of (10) with $\tau_{0}$ is any real such that $2 \tau_{0} \lambda_{M} K_{\lambda} \psi_{M}^{*} K_{e} \delta<\bar{\varepsilon}_{0}$ and $\varepsilon_{0}=\bar{\varepsilon}_{0}-2 \tau_{0} \lambda_{M} K_{\lambda} \psi_{M}^{*} K_{e} \delta$. The left side of (10) is established similarly

\section{APPENDIX C. PROOF OF THEOREM 1}

The proof is divided in two parts. First, it is shown that the mapping $e(t) \rightarrow \mid \tilde{\theta}(t) \eta(t)\rfloor$ is ISS. Then, it is shown that, in turn the mappinginverse $[\tilde{\theta}(t) \eta(t)] \rightarrow e(t)$ is ISS. The result of Theorem 1 is then obtained invoking the small gain theorem.

Part 1. Proof that the mapping $e(t) \rightarrow\left[\begin{array}{c}\tilde{\theta}(t) \\ \eta(t)\end{array}\right]$ is ISS.

Time-derivation of $V$ gives, using (15), (9a-b) and (3g):

$$
\begin{aligned}
\dot{V}= & \tilde{\theta}^{T} \dot{R}^{-1} \tilde{\theta}+2 \tilde{\theta}^{T} R^{-1} \dot{\tilde{\theta}}+2 \eta^{T} P \dot{\eta} \\
= & \tilde{\theta}^{T}\left(-R^{-1}+\lambda^{T} c^{T} c \lambda\right) \tilde{\theta}-2 \tilde{\theta}^{T}\left(\lambda^{T} c^{T} c \lambda \tilde{\theta}+\lambda^{T} c^{T} c \eta-\lambda^{T} c^{T} e\right) \\
& +2 \eta^{T} P\left(\left(A_{0}-K c\right) \eta+K e+\left(\psi_{0}(u, \operatorname{sat}(z))-\psi_{0}(u, y)\right) \theta_{0}\right) \\
\leq & -\tilde{\theta}^{T} R^{-1} \tilde{\theta}-\tilde{\theta}^{T} \lambda^{T} c^{T} c \lambda \tilde{\theta}-2 \tilde{\theta}^{T}\left(\lambda^{T} c^{T} c \eta-\lambda^{T} c^{T} e\right)-\mu\|\eta\|^{2} \\
& +2 \eta^{T} P\left(\operatorname{Ke}+\left(\psi_{0}(u, \operatorname{sat}(z))-\psi_{0}(u, y)\right) \theta_{0}\right)
\end{aligned}
$$

where we have used the fact that $2 \eta^{T} P\left(A_{0}-K c\right) \eta \leq-\mu\|\eta\|^{2}$, which is a direct consequence of (16). The last inequality further simplifies to:

$$
\begin{gathered}
\dot{V} \leq-\tilde{\theta}^{T} R^{-1} \tilde{\theta}-\tilde{\theta}^{T} \lambda^{T} c^{T} c \lambda \tilde{\theta}+\alpha\|\tilde{\theta}\|^{2}+\frac{1}{\alpha}\left\|\lambda^{T} c^{T} c \eta-\lambda^{T} c^{T} e\right\|^{2} \\
-\frac{\mu}{2}\|\eta\|^{2}+\frac{2}{\mu}\|P\|^{2}\left(\|K\|^{2}+\left\|\theta_{0}\right\|^{2}\left(\psi_{M}^{*}\right)^{2}\right) e^{2}
\end{gathered}
$$

where $\alpha>0$ is any real constant satisfying (18) and $\mu>0$ is as in (16). Note that inequality $(\mathrm{C} 1)$ is obtained using the Young inequality $\sigma$ twice, once with $\varsigma=\alpha$ and once with $\varsigma=\mu / 2$. Also note that, the last term on the right side of $(\mathrm{C} 1)$ is obtained using also the fact that:

$$
\begin{aligned}
\left\|\psi_{0}(u, \operatorname{sat}(z))-\psi_{0}(u, y)\right\| & \leq \psi_{M}^{*}|\operatorname{sat}(z)-y| \leq \psi_{M}^{*}|\operatorname{sat}(z)-\operatorname{sat}(y)| \\
& \leq \psi_{M}^{*}|e|
\end{aligned}
$$

because $\operatorname{sat}(y)=y$, with $\psi_{M}^{*}$ as in (20). Using (11) and (18), inequality (C1) develops further as follows:

$$
\begin{aligned}
\dot{V} \leq & -\frac{1}{2} \tilde{\theta}^{T} R^{-1} \tilde{\theta}+\frac{2}{\alpha}\left\|\lambda^{T} c^{T} c\right\|^{2}\|\eta\|^{2}+\frac{2}{\alpha}\left\|\lambda^{T} c^{T}\right\|^{2}|e|^{2}-\frac{\mu}{2}\|\eta\|^{2} \\
& +\frac{2}{\mu}\|P\|^{2}\left(\|K\|^{2}+\left\|\theta_{0}\right\|^{2}\left(\psi_{M}^{*}\right)^{2}\right) e^{2} \\
\leq & -\frac{1}{2} \tilde{\theta}^{T} R^{-1} \tilde{\theta}-\left(\frac{\mu}{2}-\frac{2}{\alpha}\left\|\lambda^{T} c^{T} c\right\|^{2}\right)\|\eta\|^{2} \\
& +\left(\frac{2}{\mu}\|P\|^{2}\left(\|K\|^{2}+\left\|\theta_{0}\right\|^{2}\left(\psi_{M}^{*}\right)^{2}\right)+\frac{2}{\alpha}\left\|\lambda^{T} c^{T}\right\|^{2}\right) e^{2}
\end{aligned}
$$

It is readily checked that $\left|\lambda^{T} c^{T} c\right| \leq\left|\lambda\|\mid c\|^{2} \leq \lambda_{M}\|c\|^{2}\right.$, where $\lambda_{M}$ is as in (6). By (17), $\mu$ is selected such that $\frac{\mu}{2}>\frac{2 \lambda_{M}^{2}\|c\|^{4}}{\alpha}$. Then one has, for all $s \geq 0$ :

$\frac{\mu}{2}-\frac{2}{\alpha}\left\|\lambda^{T} c^{T} c\right\|^{2}>\frac{\mu}{2}-\frac{2 \lambda_{M}^{2}\|c\|^{4}}{\alpha}>0$.

Then, it follows from $(\mathrm{C} 2)$ that:

$\dot{V} \leq-\sigma_{0} V+\sigma_{1} e^{2}$

with $\sigma_{0}$ and $\sigma_{1}$ as in (22a). Integrating the above inequality implies, for all $0 \leq t_{0}<t$ :

$V(t) \leq e^{-\sigma_{0}\left(t-t_{0}\right)} V\left(t_{0}\right)+\sigma_{1} \int_{t_{0}}^{t} e^{-\sigma_{0}(t-s)} e^{2}(s) d s$

Given any scalar $\sigma$ such that $0<\sigma<\sigma_{0} / 2$, it follows multiplying both sides of (C3) by $e^{\sigma t}$ :

$$
\begin{aligned}
e^{\sigma t} V(t) & \leq e^{-\left(\sigma_{0}-\sigma\right) t} e^{\sigma_{0} t_{0}} V\left(t_{0}\right)+\sigma_{1} e^{\sigma t} \int_{t_{0}}^{t} e^{-\sigma_{0}(t-s)} e^{2}(s) d s \\
& \leq M_{0}+\sigma_{1} e^{\sigma t} \int_{t_{0}}^{t} e^{-\sigma_{0}(t-s)} e^{2}(s) d s
\end{aligned}
$$

with $M_{0}=e^{\sigma_{0} t_{0}} V\left(t_{0}\right)$, using the fact that $e^{-\left(\sigma_{0}-\sigma\right) t}<1$. Inequality (C4) implies successively:

$$
\begin{aligned}
e^{\sigma t} V(t) & \leq M_{0}+\sigma_{1} e^{\sigma t} \int_{t_{0}}^{t} e^{-\sigma_{0}(t-s)} e^{-\sigma s} e^{\sigma s} e^{2}(s) d s \\
& \leq M_{0}+\sigma_{1} e^{\left(\sigma-\sigma_{0}\right) t} \int_{t_{0}}^{t} e^{-\left(\sigma-\sigma_{0}\right) s} e^{\sigma s} e^{2}(s) d s \\
& \leq M_{0}+\sigma_{1} e^{\left(\sigma-\sigma_{0}\right) t}\left(\int_{t_{0}}^{t} e^{-\left(\sigma-\sigma_{0}\right) s} d s\right) \sup _{t_{0} \leq s \leq t}\left(e^{\sigma s} e^{2}(s)\right) \\
& \leq M_{0}+\frac{\sigma_{1}}{\sigma_{0}-\sigma}\left(1-e^{\left(\sigma-\sigma_{0}\right)\left(t-t_{0}\right)}\right) \sup _{t_{0} \leq s \leq t}\left(e^{\sigma s} e^{2}(s)\right) \\
& \leq M_{0}+\frac{\sigma_{1}}{\sigma_{0}-\sigma} \sup _{t_{0} \leq s \leq t}\left(e^{\sigma s} e^{2}(s)\right) \leq M_{0}+\frac{2 \sigma_{1}}{\sigma_{0}} \sup _{t_{0} \leq s \leq t}\left(e^{\sigma s} e^{2}(s)\right)
\end{aligned}
$$

Taking root-squares of both sides of the last inequality gives:

$e^{\sigma / 2} \sqrt{V(t)} \leq \sqrt{M_{0}}+\sqrt{\frac{2 \sigma_{1}}{\sigma_{0}}} \sup _{t_{0} \leq s \leq t}\left(e^{\sigma / 2}|e(s)|\right)$

On the other hand, it readily follows from (15):

$V=\tilde{\theta}^{T} R^{-1} \tilde{\theta}+\eta^{T} P \eta \geq \sigma_{2}\left(\|\tilde{\theta}\|^{2}+\|\eta\|^{2}\right)$

with $\sigma_{2}$ as in (22b). Taking the root square of both sides of the above inequality, one immediately obtains $\sqrt{V} \geq \sqrt{\sigma_{2}}\left\|\left[\tilde{\theta}^{T} \eta^{T}\right]\right\|$ which, together with (C5), yields:

$\left\|\left[\begin{array}{ll}\tilde{\theta} & \eta\end{array}\right]\right\| e^{\sigma / 2} \leq \sqrt{\frac{M_{0}}{\sigma_{2}}}+\sqrt{\frac{2 \sigma_{1}}{\sigma_{0} \sigma_{2}}} \sup _{t_{0} \leq s \leq t}\left(e^{\sigma / 2}|e(s)|\right)$

Clearly, the right side of this inequality is increasing. Then, one has:

$\sup _{t_{0} \leq s \leq t}\left(\left\|\left[\tilde{\theta}^{T}(s) \eta^{T}(s)\right]\right\| e^{\sigma s / 2}\right) \leq M_{1}+\gamma \sup _{t_{0} \leq s \leq t}\left(e^{\sigma s / 2}|e(s)|\right)$

with $M_{1}=\sqrt{e^{\sigma_{0} t_{0}} V\left(t_{0}\right) / \sigma_{2}}$ and $\gamma$ as in (21), which establishes Part 1 .

Part 2. Proof of Theorem 1.

To establish Theorem 1, the expression (B6) (see Appendix B) giving $e(t)=z(t)-y(t)$ is rewritten as follows, for $k \in \mathbf{N}$ and $t \in\left[t_{k}, t_{k+1}\right)$ :

$e(t)=\int_{t_{k}}^{t} c A_{0}\left(P_{x}\left(\hat{x}(s)-P_{x}(x(s))\right) d s\right.$ 


$$
\begin{aligned}
& \quad+\int_{t_{k}}^{t} c\left[\psi_{0}(u(s), \operatorname{sat}(z(s))) \psi_{1}(u(s))\right]\left(P_{\theta}(\hat{\theta}(s))-P_{\theta}(\theta)\right) \\
& \quad+\int_{t_{k}}^{t} c\left[\psi_{0}(u(s), \operatorname{sat}(z(s)))-\psi_{0}(u(s), \operatorname{sat}(y(s))) 0\right] P_{\theta}(\theta) d s \\
& =\int_{t_{k}}^{t} c A_{0}\left(P_{x}\left(\hat{x}(s)-P_{x}(x(s))\right) d s\right. \\
& \quad+\int_{t_{k}}^{t} c\left[\psi_{0}(u(s), \operatorname{sat}(z(s))) \psi_{1}(u(s))\right]\left(P_{\theta}(\hat{\theta}(s))-P_{\theta}(\theta)\right) \\
& +\int_{t_{k}}^{t} c\left(\psi _ { 0 } \left(u(s), \operatorname{sat}(z(s))-\psi_{0}(u(s), \operatorname{sat}(y(s))) \theta_{0} d s\right.\right.
\end{aligned}
$$

where we have used the fact that $x(s)=P_{x}(x(s)), \theta=P_{\theta}(\theta)$ and $y(s)=\operatorname{sat}(y(s))$, due to (4) and (5a-b). By (8), $\eta+\lambda \tilde{\theta}$ can be substituted to $\tilde{x}$ on the right side of (C7). Then, taking absolute value of both sides of (C7) and multiplying the obtained inequality by $e^{\sigma / 2}$ one gets, using the inequalities $\left\|P_{x}(\hat{x}(s))-P_{x}(x(s))\right\| \leq\|\tilde{x}(s)\|$ and $\left\|P_{\theta}(\hat{\theta}(s))-P_{\theta}(\theta)\right\| \leq\|\tilde{\theta}\|:$

$$
\begin{gathered}
e^{\sigma / 2}|e(t)| \leq e^{\sigma / 2} \int_{t_{k}}^{t}\left\|c A_{0}\right\|\|\eta+\lambda \tilde{\theta}\| d s \\
+e^{\sigma / 2} \int_{t_{k}}^{t}\|c\|\left\|\left[\psi_{0}(u(s), \operatorname{sat}(z(s))) \psi_{1}(u(s))\right]\right\|\|\tilde{\theta}(s)\| d s \\
+e^{\sigma / 2} \int_{t_{k}}^{t}\|c\| \| \psi_{0}(u(s), \operatorname{sat}(z(s)))-\psi_{0}\left(u(s), \operatorname{sat}(y(s))\|\| \theta_{0} \| d s\right. \\
\leq e^{\sigma / 2} \int_{t_{k}}^{t}\left\|c A_{0}\right\|\|\eta\| d s+\max \left(\left\|c A_{0}\right\| \lambda_{M},\|c\| \psi_{M}\right)_{0} e^{\sigma / 2}\|\tilde{\theta}(s)\| d s \\
\quad+\|c\|\left\|\theta_{0}\right\| \psi_{M}^{*} e^{\sigma / 2} \int_{t_{k}}^{t}|e(s)| d s \\
\leq e^{\sigma / 2} M \int_{t_{k}}^{t}\|[\|\tilde{\theta}(s)\|\|\eta(s)\|]\| d s+\|c\|\left\|\theta_{0}\right\| \psi_{M}^{*} e^{\sigma / 2} \int_{t_{k}}^{t}|e(s)| d s
\end{gathered}
$$

for all $t \in\left[t_{k}, t_{k+1}\right)$, with $M=\max \left(\left\|c A_{0}\right\|,\left\|c A_{0}\right\| \lambda_{M},\|c\| \psi_{M}\right)$ and $\psi_{M}^{*}$ as in (20). Noticing that $\|\left[\|\tilde{\theta}\|\|\eta\|\|\|^{2}=\left\|\left[\begin{array}{ll}\tilde{\theta}^{T} & \eta^{T}\end{array}\right]\right\|^{2}\right.$, inequality (C8) rewrites:

$$
\begin{aligned}
& e^{\sigma / 2}|e(t)| \leq e^{\sigma / 2} M \int_{t_{k}}^{t}\left\|\left[\tilde{\theta}^{T}(s) \eta^{T}(s)\right]\right\| d s+\|c\|\left\|\theta_{0}\right\| \psi_{M}^{*} e^{\sigma / 2} \int_{t_{k}}^{t}|e(s)| d s \\
& \leq e^{\sigma / 2} M \int_{t_{k}}^{t} e^{-\sigma / 2} e^{\sigma / 2}\left\|\left[\tilde{\theta}^{T}(s) \eta^{T}(s)\right]\right\| d s \\
& +\|c\|\left\|\theta_{0}\right\| \psi_{M}^{*} e^{\sigma / 2} \int_{t_{k}}^{t} e^{-\sigma s / 2} e^{\sigma s / 2}|e(s)| d s \\
& \leq e^{\sigma / 2} M\left(\int_{t_{k}}^{t} e^{-\sigma / 2} d s\right) \sup _{t_{k} \leq s \leq t}\left(e^{\sigma / 2}\left\|\left[\tilde{\theta}^{T}(s) \eta^{T}(s)\right]\right\|\right) \\
& +\|c\|\left\|\theta_{0}\right\| \psi_{M}^{*} e^{\sigma / 2}\left(\int_{t_{k}}^{t} e^{-\sigma / 2} d s\right) \sup _{t_{k} \leq s \leq t}\left(e^{\sigma / 2}|e(s)|\right)
\end{aligned}
$$

It is readily checked that $0<\int_{t_{k}}^{t} e^{-\sigma s / 2} d s \leq \tau e^{-\sigma_{k} / 2}$. Then, one gets from (C9):

$$
\begin{aligned}
e^{\sigma / 2}|e(t)| \leq & \tau e^{\sigma\left(t-t_{k}\right) / 2} M \sup _{t_{k} \leq s \leq t}\left(e^{\sigma / 2}\left\|\left[\tilde{\theta}^{T}(s) \eta^{T}(s)\right]\right\|\right) \\
& +\tau e^{\sigma\left(t-t_{k}\right) / 2}\|c\|\left\|\theta_{0}\right\| \psi_{M}^{*} \sup _{t_{k} \leq s \leq t}\left(e^{\sigma s / 2}|e(s)|\right) \\
\leq & \tau e^{\sigma \tau / 2} M \sup _{t_{k} \leq s \leq t}\left(e^{\sigma / 2}\left\|\left[\tilde{\theta}^{T}(s) \eta^{T}(s)\right]\right\|\right) \\
& +\tau e^{\sigma / 2}\|c\|\left\|\theta_{0}\right\| \psi_{M}^{*} \sup _{t_{k} \leq s \leq t}\left(e^{\sigma s / 2}|e(s)|\right) \\
\leq & \tau e^{\sigma \tau / 2} M \sup _{t_{0} \leq s \leq t}\left(e^{\sigma / 2}\left\|\left[\tilde{\theta}^{T}(s) \eta^{T}(s)\right]\right\|\right) \\
& +\tau e^{\sigma \tau / 2}\|c\|\left\|\theta_{0}\right\| \psi_{M}^{*} \sup _{t_{0} \leq s \leq t}\left(e^{\sigma s / 2}|e(s)|\right)
\end{aligned}
$$

where we have used the inequalities $0<t-t_{k}<\tau$, with $\tau=\sup _{k}\left(t_{k}-t_{k-1}\right)$, and the fact that $t_{k}$ is an increasing sequence. It is readily seen that, the right side of inequality $(\mathrm{C} 10)$ is an increasing function of $t$. Then, it follows that:

$$
\sup _{t_{0} \leq s \leq t}\left(e^{\sigma / 2}|e(s)|\right) \leq M \tau e^{\sigma \tau / 2} \sup _{t_{0} \leq s \leq t}\left(e^{\sigma s / 2}\left\|\left[\tilde{\theta}^{T}(s) \eta^{T}(s)\right]\right\|\right)
$$

$$
+\|c\|\left\|\theta_{0}\right\| \psi_{M}^{*} \tau e^{\sigma \tau / 2} \sup _{t_{0} \leq s \leq t}\left(e^{\sigma s / 2}|e(s)|\right)
$$

Now, by letting $\tau$ be sufficiently small so that:

$\|c\|\left\|\theta_{0}\right\| \psi_{M}^{*} \tau e^{\sigma \tau / 2}<1$,

one gets from $(\mathrm{C} 11)$ :

$\sup _{t_{0} \leq s \leq t}\left(e^{\sigma t / 2}|e(t)|\right) \leq \frac{M \tau e^{\sigma \tau / 2}}{1-\|c\|\left\|\theta_{0}\right\| \psi_{M}^{*} \tau e^{\sigma \tau / 2}} \sup _{t_{0} \leq s \leq t}\left(e^{\sigma s / 2}\left\|\left[\tilde{\theta}^{T}(s) \eta^{T}(s)\right]\right\|\right)(\mathrm{C} 13)$ Substituting the right side of (C13) to $\sup _{t_{0} \leq s \leq t}\left(e^{\sigma / 2}|e(s)|\right)$ in (C6) gives: $\left.\sup _{t_{0} \leq s \leq t}\left\|\left[\tilde{\theta}^{T}(s) \eta^{T}(s)\right]\right\| e^{\sigma / 2}\right)$

$$
\leq M_{1}+\frac{\gamma M \tau e^{\sigma \tau / 2}}{1-\|c\|\left\|\theta_{0}\right\| \psi_{M}^{*} \tau e^{\sigma \tau / 2}} \sup _{t_{0} \leq s \leq t}\left(e^{\sigma s / 2}\left\|\left[\tilde{\theta}^{T}(s) \eta^{T}(s)\right]\right\|\right)
$$

Let $\tau$ be sufficiently small so that, in addition to (C12), the following inequality holds:

$$
\frac{\gamma M \tau e^{\sigma \tau / 2}}{1-\|c\|\left\|\theta_{0}\right\| \psi_{M}^{*} \tau e^{\sigma \tau / 2}}<1
$$

Then, (C14) yields:

$$
\sup _{t_{0} \leq s \leq t}\left(\left\|\left[\tilde{\theta}^{T}(s) \eta^{T}(s)\right]\right\| e^{\sigma s / 2}\right) \leq M_{1} \frac{1-\|c\|\left\|\theta_{0}\right\| \psi_{M}^{*} \tau e^{\sigma \tau / 2}}{1-\|c\|\left\|\theta_{0}\right\| \psi_{M}^{*} \tau e^{\sigma \tau / 2}-\gamma M \tau e^{\sigma \tau / 2}}
$$

This implies that:

$\left\|\left[\tilde{\theta}^{T}(s) \eta^{T}(s)\right]\right\| e^{\sigma / 2} \leq M_{1} \frac{1-\|c\|\left\|\theta_{0}\right\| \psi_{M}^{*} \tau e^{\sigma \tau / 2}}{1-\|c\|\left\|\theta_{0}\right\| \psi_{M}^{*} \tau e^{\sigma \tau / 2}-\gamma M \tau e^{\sigma \tau / 2}}$

which ends the proof

\section{REFERENCES}

[1] Ahmed-Ali T., R. Postoyan, F. Lamnabhi-Lagarrigue (2009). Continuous discrete adaptive observers for state affine systems. Automatica, vol. 45, pp. 2986-2990.

[2] Bastin G., Gevers M. (1988). Stable adaptive observers for nonlinear time-varying systems. IEEE Transactions on Automatic Control; vol. 33, pp. 650-658.

[3] Besançon G. Nonlinear Observers and Applications. Springer, 2007.

[4] Besançon G., J. De León-Morales, O. Huerta-Guevara (2006). On adaptive observers for state affine systems. International Journal of Control, vol. 79 (6), pp. 581-591.

[5] Hann C.A.B. and T. Ahmed-Ali (2012). Continuous adaptive observer for state affine sampled-data systems. Int. J. Robust and Nonlinear Control, vol. 24 (4), pp. 669-681.

[6] Karafyllis I., C. Kravaris (2009). From continuous-time design to sampled-data design of observers. IEEE Trans. on Aut. Control, vol. 54 (9), pp. 2169-2174.

[7] Marino R, P. Tomei (1996). Nonlinear control design: geometric, adaptive and robust. Prentice Hall, UK.

[8] Zhang Q. (2002). Adaptive observer for multiple-input-multipleoutput (mimo) linear time-varying systems. IEEE Trans. Automatic Control, vol. 47 (3), pp. 525-529. 